\title{
Dedicated Stochastic Framework for the Variability Analysis of Compressible Textile Antennas
}

\author{
Marco Rossi*, Sam Agneessens, Dries Vande Ginste, Hendrik Rogier \\ Dept. of Information Technology/iMinds \\ Ghent University \\ Ghent, Belgium \\ marco.rossi@intec.ugent.be
}

\begin{abstract}
A dedicated stochastic framework to address the impact of substrate compression on the performance of textile antennas is presented. The joint probability distribution of the height and the permittivity of a compressible substrate is experimentally estimated. Then, a generalized polynomial chaos technique accurately quantifies the effect of the random variations on both substrate parameters on the figures of merit of a dual-polarized ISM textile antenna.
\end{abstract}

\section{INTRODUCTION}

Among the challenges posed by the deployment of wearable components in critical scenarios, the effect of substrate compression on the performance of textile antennas still represents an open problem for designers. Up to now, this effect is accounted for in the design phase by checking the performance of the antenna for different values of the thickness of the substrate and by adjusting the permittivity accordingly, since the material density and, hence, its dielectric constant, increases under compression. However, this approach is not rigorous nor supported by experimental results on the variations of height and permittivity and on the correlation between them. Therefore, we present a stochastic framework that combines the measurements performed on several samples of a compressible substrate with a non-intrusive stochastic collocation method (SCM) based on generalized polynomial chaos (gPC) [1] to quantify the impact of variations in substrate permittivity and thickness on the antenna's figures of merit. An example demonstrates a speedup factor of 83 compared to Monte Carlo.

\section{StOCHASTIC FRAMEWORK FOR COMPRESSIBLE ANTENNAS}

\section{A. Stochastic Collocation Method}

Consider the textile dual-polarized probe-fed compressible ISM patch antenna shown in Fig. 1, which is designed (see Table I) to resonate at the frequency $f_{r}=2.45 \mathrm{GHz}$. Our aim is to assess the effect of stochastic variations in the substrate permittivity $\epsilon_{r}$ and height $h$ on the magnitude of the return loss $S_{11}$ at the operating frequency $f_{r}$, which is required to be lower than $0.316(-10 \mathrm{~dB})$. Therefore, we introduce a SCM and we define $\left|S_{11}\right|$ as a function of two normalized independent input random variables $x_{1}$ and $x_{2}$, corresponding to $h$ and $\epsilon_{r}$, respectively, by the following gPC expansion of total order $2 Q:$

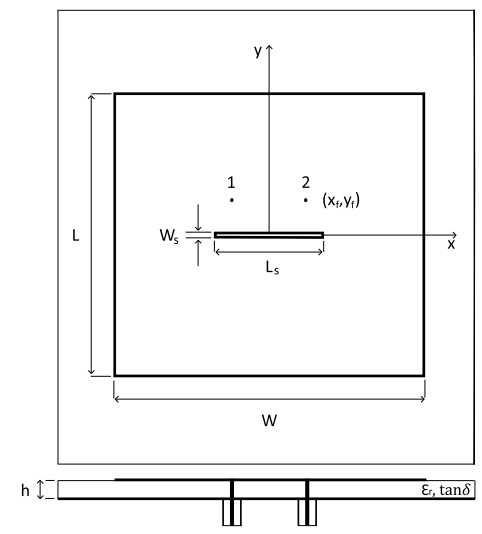

Fig. 1. Schematic of the dual polarized ISM textile antenna under study.

TABLE I

NOMINAL VALUES OF THE ANTENNA'S GEOMETRICAL PARAMETERS (FIG. 1).

\begin{tabular}{|c|c|}
\hline parameter & value \\
\hline patch length $L$ & $44.46 \mathrm{~mm}$ \\
\hline patch width $W$ & $45.32 \mathrm{~mm}$ \\
\hline slot length $L_{S}$ & $14.88 \mathrm{~mm}$ \\
\hline slot width $W_{S}$ & $1 \mathrm{~mm}$ \\
\hline feed points $\left(x_{f}, y_{f}\right)$ & $( \pm 5.7,5.7) \mathrm{mm}$ \\
\hline
\end{tabular}

$$
\left|S_{11}\right|=f\left(x_{1}, x_{2}\right)=\sum_{k_{1}=0}^{Q} \sum_{k_{2}=0}^{Q} t_{k_{1} k_{2}} \phi_{k_{1}}^{X_{1}}\left(x_{1}\right) \phi_{k_{2}}^{X_{2}}\left(x_{2}\right)
$$

where $\phi_{k_{i}}^{X_{i}}\left(x_{i}\right)$ are polynomial basis functions of degree $k_{i}$ corresponding to the $i$-th variable $(i=1,2)$. Since $x_{1}$ and $x_{2}$ are mutually independent, the polynomials $\phi_{k_{i}}^{X_{i}}\left(x_{i}\right)$ are introduced as functions of a single random variable. Moreover, they are orthonormal with respect to the following inner product:

$$
<u\left(x_{i}\right), v\left(x_{i}\right)>=\int_{\Gamma_{i}} u\left(x_{i}\right) v\left(x_{i}\right) p_{X_{i}}\left(x_{i}\right) d x_{i},
$$

where $\Gamma_{i}$ is the support of the PDF $p_{X_{i}}\left(x_{i}\right)$ of the random variable $x_{i}$, and are selected according to the Wiener-Askey scheme for commonly used PDFs [1]. Any existing correlation among the variations on the antenna's design parameters $x_{1}^{\prime}, x_{2}^{\prime}$ is related to the normalized random variables $x_{1}, x_{2}$ in (1) by

$$
[x]^{\prime}=[T][x]+[\mu],
$$


where $[T]$ is the upper triangular matrix resulting from the Cholesky decomposition of the covariance matrix $[\Sigma]$ corresponding to the joint PDF of $x_{1}^{\prime}, x_{2}^{\prime}$, whereas $[\mu]$ is the vector of the mean values of $x_{1}^{\prime}, x_{2}^{\prime}$. The coefficients $t_{k_{1} k_{2}}$ are calculated by applying Galerkin weighting to (1):

$$
t_{k_{1} k_{2}}=<f, \phi_{k_{1}}^{X_{1}} \phi_{k_{2}}^{X_{2}}>=\iint_{\Gamma} f \phi_{k_{1}}^{X_{1}} \phi_{k_{2}}^{X_{2}} P_{X_{1} X_{2}} d x_{1} d x_{2}
$$

where $\Gamma=\bigcup_{i} \Gamma_{i}$ is the $2 \mathrm{D}$ support of $P_{X_{1} X_{2}}=p_{X_{1}}\left(x_{1}\right) p_{X_{2}}\left(x_{2}\right)$. Integrals (4) are then evaluated by the following $V_{1} \times V_{2}$-point Gaussian quadrature rule:

$$
t_{k_{1} k_{2}} \approx \sum_{l_{1}=0}^{V_{1}} \sum_{l_{2}=0}^{V_{2}} w_{l_{1}} w_{l_{2}} \phi_{k_{1}}^{X_{1}}\left(x_{l_{1}}\right) \phi_{k_{2}}^{X_{2}}\left(x_{l_{2}}\right) f\left(x_{l_{1}}^{\prime}, x_{l_{2}}^{\prime}\right)
$$

where the quadrature points $x_{l_{1}}, x_{l_{2}}$ and weights $w_{l_{1}}, w_{l_{2}}$ are derived by the Golub-Welsch algorithm [2]. The values $f\left(x_{l_{1}}^{\prime}, x_{l_{2}}^{\prime}\right)$ are computed with ADS Momentum, after the quadrature points $x_{l_{1}}, x_{l_{2}}$ are modified according to (3) to embed the information about the correlation among the antenna's design parameters into the coefficients $t_{k_{1} k_{2}}$.

\section{B. Estimation of the input PDF}

A resonance-perturbation technique based on an aperturecoupled patch antenna is devised to estimate the PDF of the relative permittivity $\epsilon_{r}$ and the thickness $h$ of compressible substrates. The structure consists of two rigid planar circuit boards, containing the feed of the antenna and the conductive patch, respectively, with the material under test (MUT) in between. The structure was originally designed to resonate in the vicinity of the GPS-L1 frequency band. However, when the thickness and permittivity of the MUT change, this resonance frequency is shifted. Therefore, if the thickness of the substrate is known, the value of the measured resonance frequency can be used to estimate the permittivity of the sample. This procedure is carried out by simulating the structure in CST Microwave Studio's frequency domain solver with the measured value of thickness of the MUT and by varying the value of the permittivity of the MUT until the values of the simulated and measured resonance frequencies match.

\section{EXAMPLE}

The proposed stochastic framework is applied to the textile antenna shown in Fig. 1 [3], which is designed for a flexible closed-cell expanded rubber protective foam with height $h=3.94 \mathrm{~mm}$, permittivity $\epsilon_{r}=1.53$ and a loss tangent $\tan \delta=0.012$. 25 different samples of this material were compressed up to $70 \%$ of their nominal value of $h$ by means of a Hotronix Air Fusion Heat Press to achieve 10 different values of thickness and the corresponding values of relative permittivity were estimated. By fitting a bivariate normal distribution to the measured data, we obtained the mean values $\mu_{h}=3.36 \mathrm{~mm}$ and $\mu_{\epsilon_{r}}=1.58$ of the substrate thickness $h$ and permittivity $\epsilon_{r}$, respectively, as well as the covariance matrix $[\Sigma]$ of their joint PDF. The correlation value $\rho$ between $h$ and $\epsilon_{r}$ is found to be equal to -0.68 , thus indicating strong correlation between the two parameters.
The sets of orthonormal polynomials $\phi_{k_{1}}^{X_{1}}\left(x_{1}\right), \phi_{k_{2}}^{X_{2}}\left(x_{2}\right)$ simply comprise conventional Hermite polynomials, as the estimated input PDF is a bivariate normal distribution. The gPC expansion (1) converges for a maximum order of expansion $Q=10$. In order to compute the expansion coefficients $t_{k_{1} k_{2}}$, $(Q+1)^{2}=121$ quadrature points are evaluated, requiring 24 min (Intel i7 CPU, 16 GB RAM). Fig. 2 shows $\left|S_{11}\right|$ as a function of the variables $x_{1}$ and $x_{2}$, in a span of \pm 3 , corresponding to a normalized interval of $\pm 3 \sigma$. The limit of $0.316(-10 \mathrm{~dB})$ is indicated by means of a grey plane.

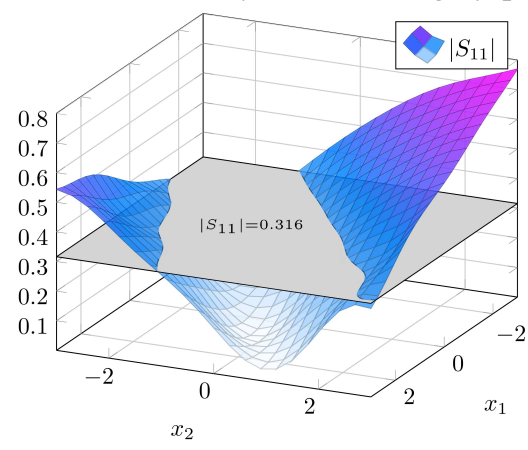

Fig. 2. $\left|S_{11}\right|$ as a function of the normalized thickness $h\left(x_{1}\right)$ and the normalized relative permittivity $\epsilon_{r}\left(x_{2}\right)$ according to SCM.

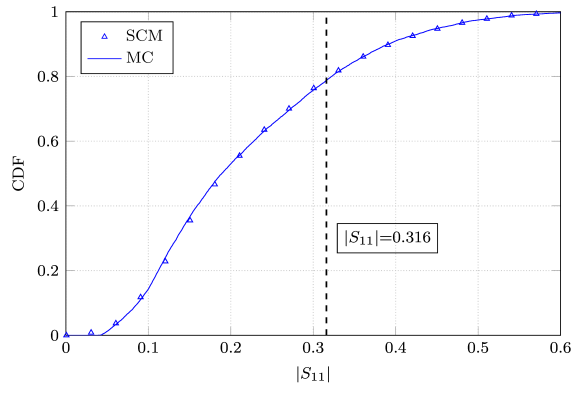

Fig. 3. Comparison between the CDFs of $\left|S_{11}\right|$ constructed with SCM and the Monte Carlo (MC) simulations.

To validate our analysis we perform a Monte Carlo simulation, evaluating 10000 realizations of $h$ and $\epsilon_{r}$ in ADS Momentum, which takes about $33 \mathrm{~h}$. The corresponding cumulative distribution functions (CDF) for $\left|S_{11}\right|$ and the one computed from the expansion (1) are shown in Fig. 3. Both CDFs are perfectly overlapping. We notice that $\left|S_{11}\right|$ is within the threshold value of 0.316 in only $79.24 \%$ of the cases.

\section{ACKNOWLEDGEMENT}

This research was partially funded by BELSPO and by the Research Foundation Flanders (FWO-Vlaanderen).

\section{REFERENCES}

[1] D. Xiu and G. E. Karniadakis, "The Wiener-Askey polynomial chaos for stochastic differential equations," SIAM Journal on Scientific Computing, vol. 24, no. 2, pp. 619-644, 2002.

[2] G. Golub and J. Welsch, "Calculation of Gauss quadrature rules," Mathematics of Computation, vol. 23, no. 106, pp. 221-230, Apr. 1969.

[3] L. Vallozzi, H. Rogier, and C. Hertleer, "Dual polarized textile patch antenna for integration into protective garments," IEEE Antennas and Wireless Propagation Letters, vol. 7, pp. 440-443, 2008. 